\title{
Magnetic resonance imaging in patients with progressive myelopathy following spinal surgery
}

\author{
ELIESER AVRAHAMI,* RINA TADMOR, $\dagger$ DAN FRANZ COHN
}

From the Departments of Radiology* and Neurology, $\ddagger$ Tel-Aviv Medical Center, and Department of Radiology, Chaim Sheba Medical Center, $\dagger$ and the Tel-Aviv University Sackler School of Medicine, Israel

SUMMARY Thirty one patients with insidious progressive myelopathy 2 to 8 years following surgery $\frac{\frac{O}{\bar{N}}}{\bar{N}}$ of the cervical spine were subjected to magnetic resonance imaging (MRI). In 15 patients operated on $\stackrel{\odot}{\circ}$ for vascular malformations or intramedullary tumours, syringomyelia and cystic lesions of the spinal $\mathrm{N}$ cord were shown. Seven of these patients also showed a combination of a recurrent tumour and spinal atrophy. Out of 16 patients who had surgery for herniated disc or spinal stenosis of the cervical spine, four had syringomyelia and 12 had spinal cord atrophy. There was no syringomyelia in the 12 patients $\vec{\omega}$ submitted to MRI prior to surgery.

Owing to the high sensitivity of magnetic resonance and its excellent contrast resolution, MRI clearly reveals the spinal cord and detects various intramedullary anomalies, including neoplasms and syringomyelia. ${ }^{1-7}$ Distinct margins of the intramedullary lesion, uniform signal intensity and isointensity with cerebrospinal fluid (CSF) in a patient with an expanded cord is consistent with a cystic lesion, syringomyelia, and the differential diagnosis of intramedullary tumour can be made with a high degree of accuracy. Poorly defined margins, inhomogeneity, and lack of isointensity with CSF suggest neoplasm. In cases where intramedullary neoplasm is associated with a syrinx, MRI may demonstrate features of both abnormalities. ${ }^{\prime}$ The differential diagnosis in patients with myelopathy following spinal surgery is very difficult, owing to the similar clinical picture in various conditions such as dural adhesions, recurrent or

Address for reprint requests: Dr E Avrahami, Department of Radiology, Tel-Aviv Medical Center, Ichilov Hospital, 64239 TelAviv, Israel.

Received 12 January 1988 and in revised form 2 May 1988. Accepted 28 June 1988 residual intramedullary tumour, residual arteriovenous malformation (AVM), recurrent or residual disc cord atrophy, or syringomyelia.

Syringomyelia refers to the presence of longitudin ally oriented cavities in the spinal cord. When the cavities extend into the brain stem, the term "syrinzgobulbia" is applicable. The cavities are surrounded by glial cells. If an ependymal lining is present, the lesion is referred to as hydromyelia. ${ }^{1-4}$

We present our experience in a group of patiente with progressive postoperative myelopathy, evaluateđ by MRI.

\section{Material and methods}

Thirty one patients with progressive myelopathy ( 7 females and 24 males), age range 11 to 64 years, underwent MRI of the spine 5 months to 8 years following spinal surgery. In 12 cases intramedullary tumour (nine astrocytoma; three ependymoma) was established by histology. In three other patients vascular malformation was confirmed by surgery. The remaining 16 patients were operated on for herniated cervical disc or stenosis of the cervical spine (table). Patients with extramedullary spinal tumours were not included in this study. Neurological examination prior to surgery revealed

Table MRI findings in 31 patients with progressive myelopathy following spinal operation

\begin{tabular}{llllcc}
\hline & Total & $\begin{array}{l}\text { Syringomyelia } \\
\text { or cyst }\end{array}$ & Atrophy & $\begin{array}{l}\text { Recurrent } \\
\text { tumour }\end{array}$ & $\begin{array}{l}\text { MRI prior } \\
\text { to surgery }\end{array}$ \\
\hline Tumour (astrocytoma or ependymoma) & 12 & 12 & 4 & 3 & 7 \\
Vascular malformation (AVM) & 3 & 3 & 12 & 1 \\
Cervical herniated disc or spinal stenosis & 16 & 4 & 16 & 3 & 12 \\
TOTAL & 31 & 19 & 3 & \\
\hline
\end{tabular}




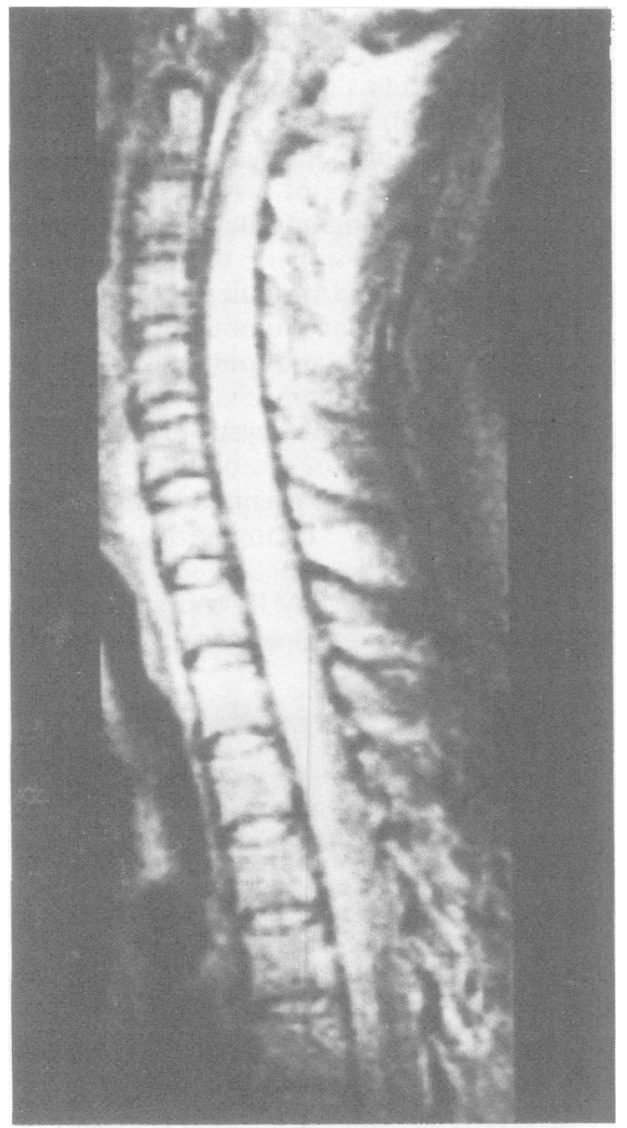

Fig 1 MRI of the cervical spine in a 21 year old male with mild progressive quadriparesis prior to surgery. $T R=$ $2000 \mathrm{~ms}, \mathrm{TE}=30 \mathrm{~ms}$. Intramedullary astrocytoma shown by diffuse widening of the spinal cord and indistinct borders, high signal and no isointensity with CSF. No cystic lesions. He developed postoperative syringomyelia similar to fig 3.

(1) Mild quadri- or paraparesis in all patients. (2) Paraesthesia or hypoaesthesia in 14 patients. (3) Pyramidal signs (spasticity of the lower extremities with positive Babinski sign) in 17 patients. (4) Slightly impaired bladder function in two patients. Clincal improvement was noted in all cases following surgery.

Prior to operation, 12 patients out of 31 included in the study underwent spinal MRI examinations, which showed no cystic intramedullary lesions (fig 1). Patients with cystic intramedullary tumours were excluded from this study.

Insidiously progressive clinical deterioration occurred in all 31 patients following surgery. There was para- or quadriparesis, with or without pyramidal signs, in all patients. Hypoaesthesia was present in 17 cases. Fasciculations of truncal or extremity muscles was present in three cases. Two cases had brain stem symptoms, such as hypoaesthesia of the face and fasciculations of the tongue. There was no dissociated sensory loss. Since the clinical symptoms of the patients were similar, a differential diagnosis between recurrent tumour, spinal cord atrophy and syringomyelia was impossible.

The patients operated on for intramedullary tumour or AVM developed neurological symptoms 5 to 20 months after surgery: the remainder becoming symptomatic 15 months to 5 years following surgery. All patients underwent spinal cord MRI examination. Scans were performed on an Elscint Gyrex S-5000 MRI Scanner, using a superconductive magnet with 0.5 Tesla field strength. Sagittal slices with $0.5 \mathrm{~mm}$ thickness were performed with $T_{1}$ and $T_{2}$ sequences using a time to repeat (TR) at 500,600 and $2000 \mathrm{~ms}$, and time to echo (TE) of 27,50 and 100 ms. The acquisition matrix was $256 /$ 256.

Twenty two patients underwent spinal CT or myelography as well, 17 of whom showed no cystic lesions or spinal cord atrophy

\section{Results}

Syringomyelia was found in all 12 patients operated on for intramedullary tumour (figs 2,3 ). Three of them had proven recurrent tumour accompanied by a cystic intramedullary lesion (fig 4). In four other patients who underwent the same operation, the syringomyelia was combined with atrophic changes of the spinal cord (fig 5). Two of the patients with intramedullary tumours have been reported on previously. ${ }^{8}$

The criteria for the diagnosis of a cystic lesion by MRI were: uniform low signal in short TR/TE, and homogeneous strong signal on $T_{2}$ with longer TR/TE; isointensity with the CSF; smooth well-defined borders, and no additional heterogenic signal in the cystic area. The accuracy of the above mentioned criteria

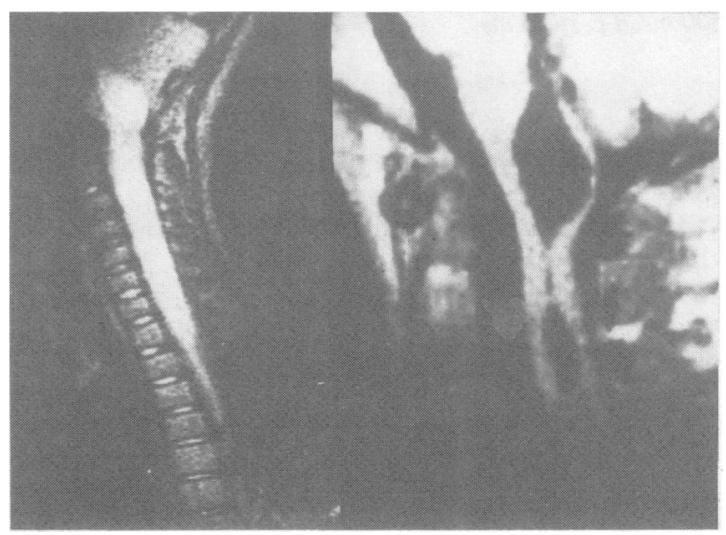

Fig 2 MRI of upper spinal cord in a 12 year old male with intramedullary astrocytoma. Left slice: prior to surgery. $T R$ $=2000 \mathrm{~ms}, T E=100 \mathrm{~ms}$. Diffuse widening of the spinal cord and indistinct borders. Strong $T_{2}$ signal of the cord from $C_{1}$ to $T_{2}$. No cyst. Right slice: same area post surgery. $T R=$ $500 \mathrm{~ms}, T E=25 \mathrm{~ms}$. Eccentric cystic lesion of the medulla and $C_{1}$. Smaller cyst at $C_{2}-C_{3}$ level. Isointensity with $C S F$. 


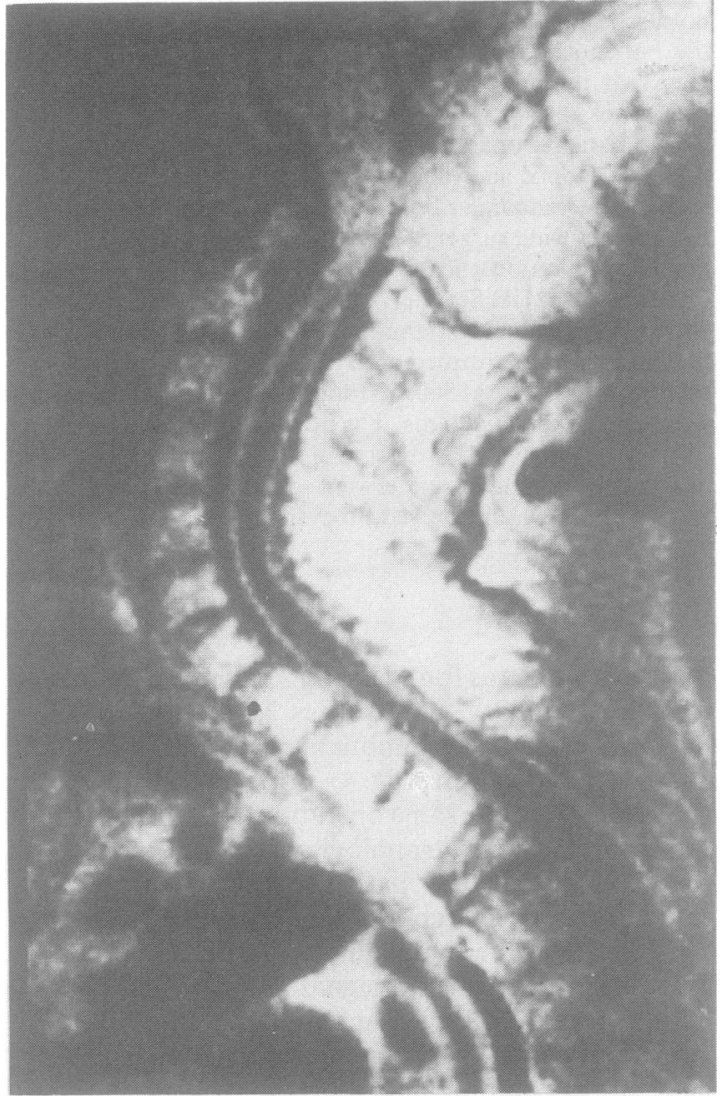

Fig 3 Holocord syringomyelia 5 months following surgery for intramedullary ependymoma in a 17 year old male. $T R=$ $500 \mathrm{~ms}, T E=50 \mathrm{~ms}$.

should be $85 \% .^{1}$ Lack of isointensity of the signals with the CSF, indistinct borders and additional signals in the cystic area are in favour of a tumour.

Holocord syringomyelia was demonstrated in all three patients with spinal AVMs 23 to 31 months following surgery and the phenomenon of transverse "septa" was observed in these patients (fig 6). There was an atrophic narrow spinal cord with sharp but irregular borders in 12 patients who underwent surgery for spinal stenosis or herniated cervical disc (fig 7). In four other patients who underwent similar surgery, syringomyelia was present in the operated area (fig 8).

\section{Discussion}

MRI is the procedure of choice for demonstration of spinal cord lesions, including spinal cord atrophy, cystic intramedullary lesions, and tumours.
Prior to surgery there was no evidence of spinal cord $\bar{k}$ atrophy or cystic intramedullary lesions in ouf subjects. All patients developed slowly progressive postoperative myelopathy with similar clinical symp气 toms. The main clinical presentation was para- of quadriparesis. Symptoms of dissociated sensory loss were absent, and syringomyelia was not suspected prior to MRI. Progression of the myelopathy was. considerably more rapid in patients who underwen $\vec{E}$ surgery for intramedullary tumour or AVM than in those with herniated disc and spinal stenosis.

The diagnosis of syringomyelia should be made as early as possible. Severe cystic lesions can be associated with mild motor disability, but irreversibles clinical deterioration may occur during the progres sion of the myelopathy. ${ }^{9}$ Draining of the cyst is the indicated treatment. ${ }^{1011}$

It is important to repeat MRI routinely in patients $\vec{\rho}$ who have undergone spinal surgery. We believe that

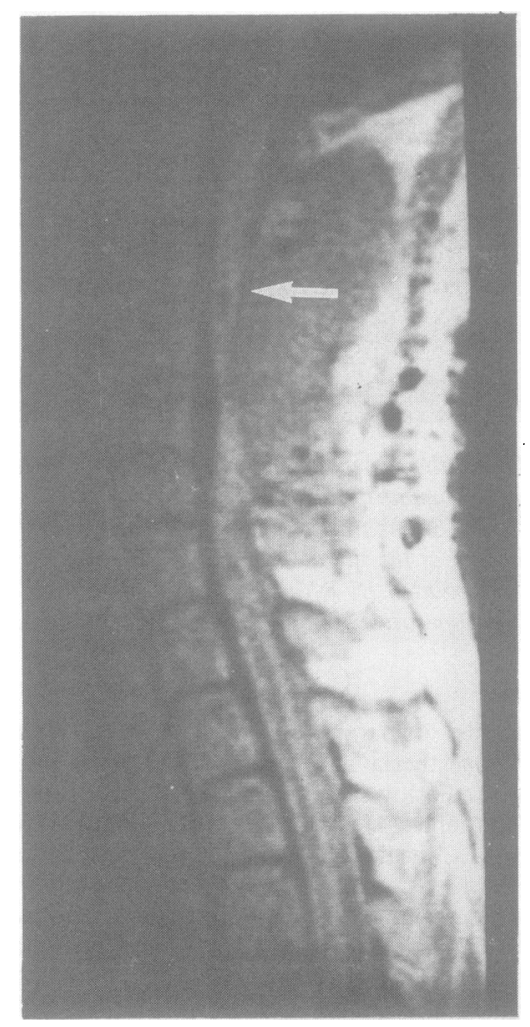

Fig 4 MRI of a 17 year old female 14 months post surgery for intramedullary ependymoma $C_{4}-C_{7}$ and progressive quadriparesis. $T R=500 \mathrm{~ms}, T E=25 \mathrm{~ms}$. Narrow spinal cord at level $C_{5}-C_{6}$ compatible with atrophy and a small intramedullary cyst (arrow). Syringomyelia below the atrophic area. 


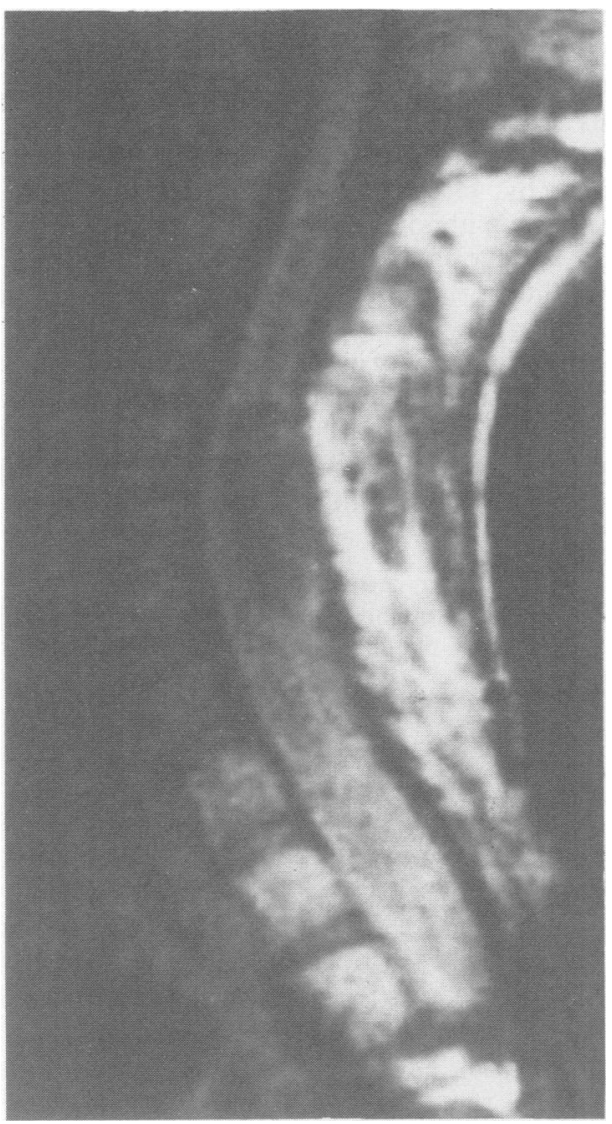

Fig 5 Recurrent intramedullary astrocytoma in a 27 year old male with progressive spastic quadriparesis, 17 months post surgery. $T R=2000 \mathrm{~ms}, T E=27 \mathrm{~ms}$. Widened spinal cord with indistinct borders and no isointensity with CSF. A cyst at $C_{6}-T_{1}$.

early intramedullary cystic lesions may be revealed by MRI prior to the onset of myelopathy. We consider that at the early postoperative stage the spinal cord cyst might be asymptomatic. Patients operated on for intramedullary tumour or AVM became symptomatic 5 to 20 months following surgery. The cystic intramedullary lesions of these patients were probably asymptomatic 1 or 2 months prior to MRI. We therefore recommend that follow-up MRI at 3 month intervals should be the rule for patients operated on for intramedullary tumours and AVMs of the spine.

Syringomyelia following surgery of spinal AVM has been reported. ${ }^{12}$ All three of our patients with this condition had holocord cystic enlargement with transverse septa (fig 6). The septa are consistent with saccular spinal dilatation. ${ }^{10}$ Less typical was the atro- phy following surgery for intramedullary tumour (table). It was always combined with cystic spinal dilatation which was demonstrated on a different level. The MRI signs of spinal cord atrophy are a narrow spinal cord with well defined but irregular borders in the operated area. Patients operated on for disc disease or spinal stenosis developed spinal pathology more slowly than those operated on for intramedullary tumour or AVM. They became symptomatic 15 months to 5 years following surgery. Surgery improved the clinical condition in all of them and they were asymptomatic in the early postoperative period. Persistent spinal cord lesion prior to surgery was clinically excluded in 12 patients; in the remaining four patients preoperative MRI examination demonstrated normal spinal cord (fig 9).

The clinical material at our disposal was insufficient to draw any conclusions regarding the dependency of

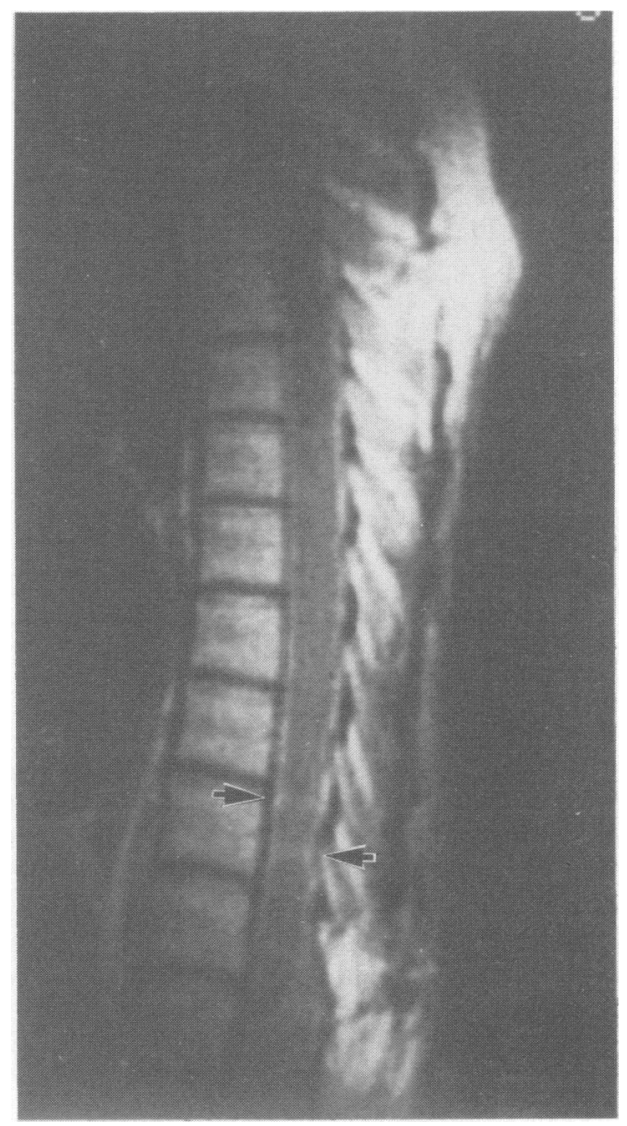

Fig 6 Slowly progressive paraparesis in a 27 year old female operated on for AVM 25 months before. TR $=2000 \mathrm{~ms}, T E$ $=27 \mathrm{~ms}$. Severe syringomyelia. Note the transverse "septa" (arrows). 


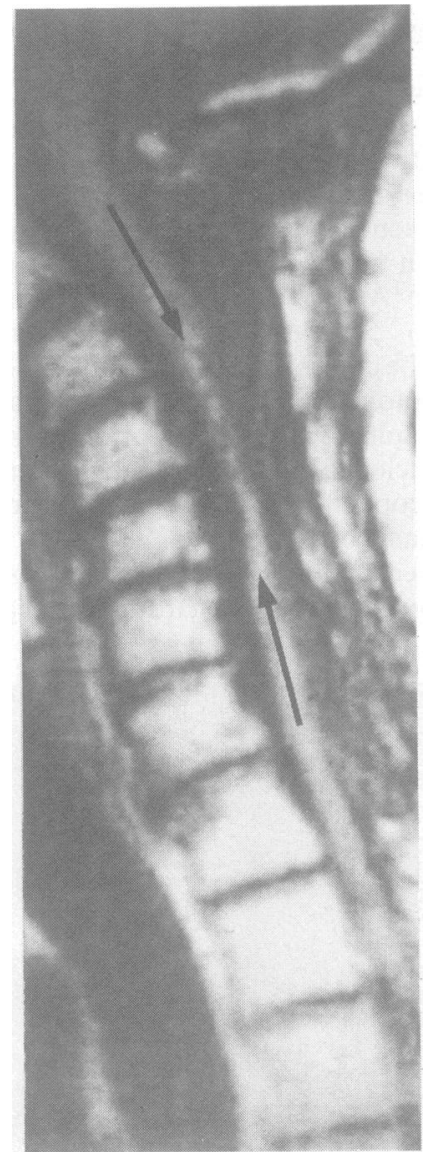

Fig 7 Spinal cord atrophy. Very slowly progressive quadriparesis in a 57 year old male who underwent surgery for spinal stenosis 4 years before. Spinal cord is narrow at $C_{4}-C_{6}$ with smooth but irregular borders (arrows). $T R=$ $500 \mathrm{~ms}, T E=27 \mathrm{~ms}$.

postoperative spinal cord lesions on the surgical approach (anterior or posterior). Our assumption presented in two preliminary reported $\operatorname{cases}^{8}$ that surgery of intramedullary tumours by Cavitron Ultrasonic Aspirator (CUSA) is responsible for the cystic dilatation of the spinal cord should be reconsidered. It is possible that any form of spinal surgery, regardless of technique, may be followed by this condition. In cases with recurrent cystic spinal cord tumour, the lesion itself may be sufficient for the development of intramedullary activities.

The mechanism of postoperative cavities remains unclear. It is most likely to be similar to that of posttraumatic syringohydromyelia. The insidious progression of clinical symptomatology reflects the slow development of the intramedullary cyst. CSF pulsations into the central canal associated with lack of $Z$ drainage through the Virchow-Robin perivascular spaces probably result in the cystic enlargement. The perivascular spaces of the perforating vessels may be blocked by bleeding or postoperative arachnoid adhesions or abnormal vessels. ${ }^{813}$ No pre-operative reason for syringomyelia, such as pathology in the floor of the fourth ventricle with herniation, was found in our cases.

The development of cystic intramedullary lesions or spinal cord atrophy may occur in patients following surgery of intradural or epidural mass lesions and in those conditions when postoperative myelopathy is suspected, MRI offers a definitive diagnosis.

\section{References}

1 Williams AL, Houghton VM, Pojunas RW, Daniels DL, $\vec{\circ}$ Kilgore DP. Differentiation of intramedullary neo-

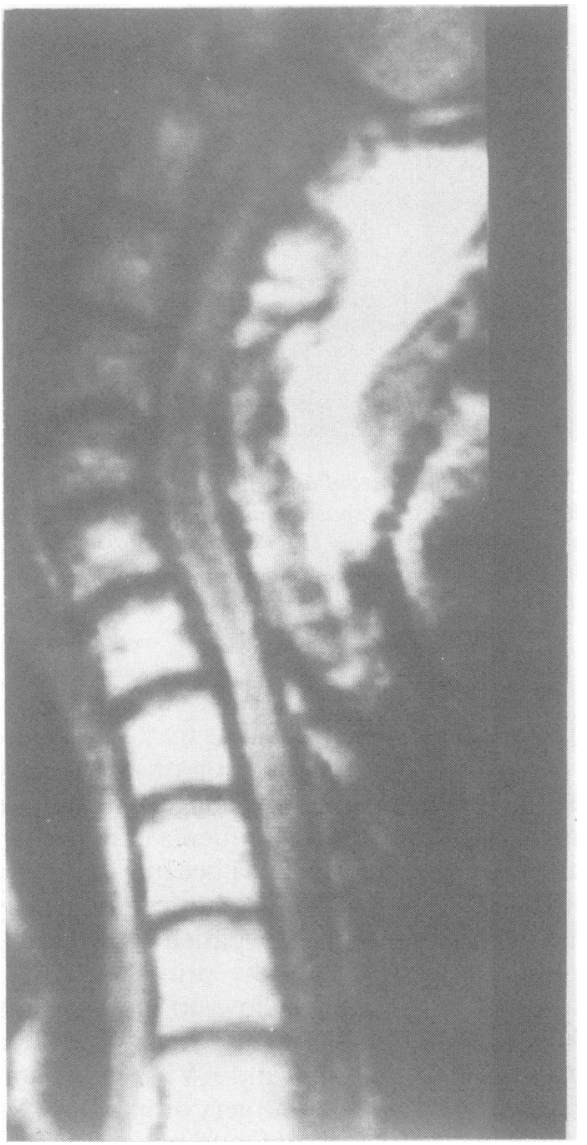

Fig 8 Syringomyelia at $C_{3}-C_{7}$ level in a 59 year old female with progressive tetraparesis 3 years after operation for $a$ herniated disc $C_{4}-C_{5} . T R=500 \mathrm{~ms}, T E=27 \mathrm{~ms}$. 


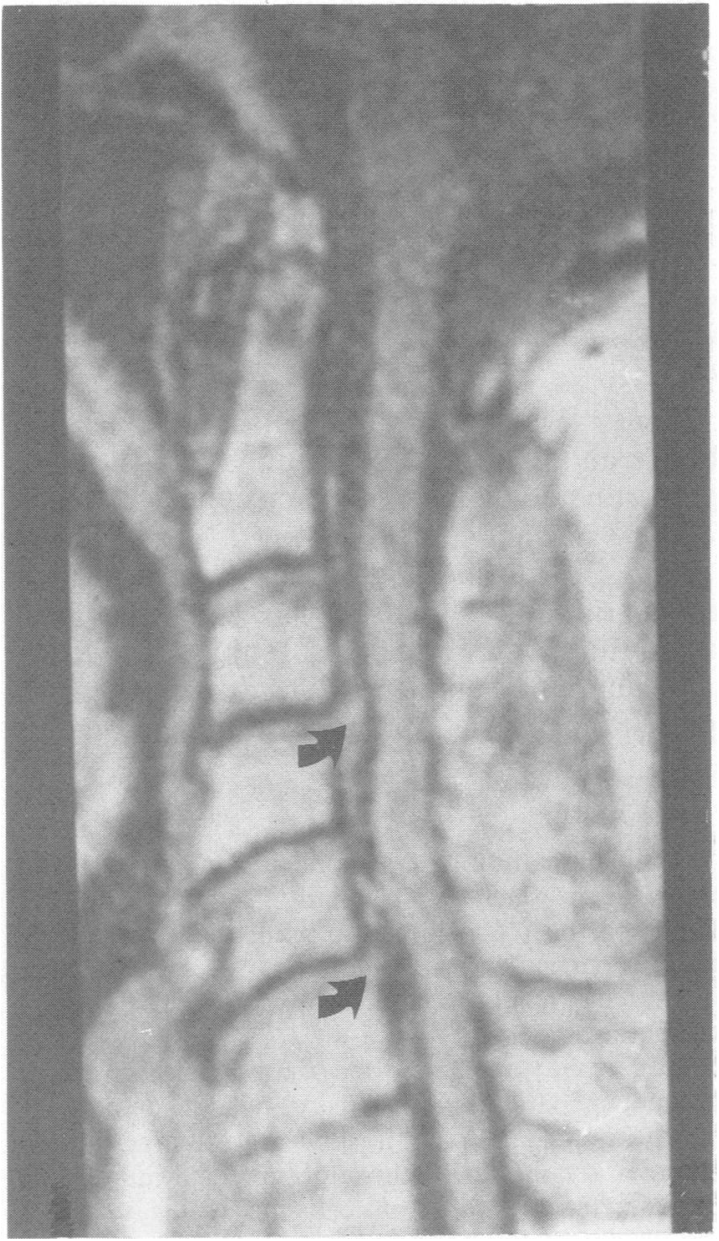

Fig $9 M R I$ of cervical spine prior to surgery of a 56 year old male. Posterior herniation of the intervertebral discs at levels $C_{3}-C_{4}$ and $C_{5}-C_{6}$ with pressure on the spinal cord (arrows). No atrophy is present. Four years after surgery, spinal cord atrophy appeared (see fig 7). plasms and cysts by MR. AJR 1987;149:159-64.

2 Pojunas K, Williams AL, Daniels DL, Houghton VM. Syringomyelia and hydromyelia: magnetic resonance evaluation. Radiology 1984;153:679-83.

3 Sherman IL, Citrin CM, Barkovich AJ. MR imaging of syringobulbia. J Comput Tomogr 1987;11:407-11.

4 Lee BCP, Zimmerman RD, Manning JJ, Deck MDF. MR imaging of syringomyelia and hydromyelia. $A J N R$ 1985;6:221-8.

5 Sherman IL, Barkovich AJ, Citrin CM. The MRI appearance of syringomyelia: new observations. AJNR 1986;7:985-95.

6 Yeates A, Brant-Zawadski M, Norman D, Kaufman L, Crooks LE, Newton TH. Nuclear magnetic imaging of syringomyelia. AJNR 1983;4:234-7.

7 Tadmor R, New PEJ, Ghoukimos G, Edelman RR, Brady TJ. Magnetic resonance imaging of the thoracical spinal cord and spine with surface coils. Acta Radiologica Supplement. XIII Symposium Neuroradiologicum, Stockholm, June 1986;369:23-8.

8 Avrahami E, Herskowitz PI, Cohn DF. Magnetic resonance imaging of syringomyelia occurring after operation of spinal intramedullary tumors by Cavitron ultrasonic aspirator. $B r J$ Radiol 1987;60:701-2.

9 Epstein F, Epstein N. Surgical treatment of spinal cord astrocytomas of childhood. A series of 19 patients. $J$ Neurosurg 1982;57:682-5.

10 Gardner WJ. Hydrodynamic mechanism of syringomyelia: its relationship to myelocele. $J$ Neurol Neurosurg Psychiatry 1965;28:247-59.

11 Pearless SJ, Durward AJ. Management of syringomyelia: a pathophysiological approach. Clin Neurosurg 1982; 30:531-76.

12 Doppmann JL, Di Chiro G, Dwyer AJ, Frank JL, Oldfield EH. Magnetic resonance imaging of spinal arteriovenous malformations. $J$ Neurosurg 1987;66: 830-4.

13 Barkovich AJ, Sherman IL, Citrin CM, Franz G, Wippold LL. MR of postoperative syringomyelia. AJNR 1987;8:319-27. 